\title{
Inequality in the Universe, Imaginary Numbers and a Brief Solution to $P=N P$ ? Problem
}

\begin{abstract}
Mesut KAVAK*
While I was working about some basic physical phenomena, I discovered some geometric relations that also interest mathematics [1]. In this work, I applied the rules I have been proven to $\mathrm{P}=\mathrm{NP}$ ? problem over impossibility of perpendicularity in the universe. It also brings extremely interesting results out like imaginary numbers which are known as real numbers currently. Also it seems that Euclidean Geometry is impossible. The actual geometry is Riemann Geometry and complex numbers are real.
\end{abstract}

\section{Introduction}

There exist many exact proofs about the rules of right triangle in abstract math; but what are the actual geometry and mechanism in real physical medium and for real math? What an undertaking can give a triangle about existence to understand it? Actually, it is able to explain everything.

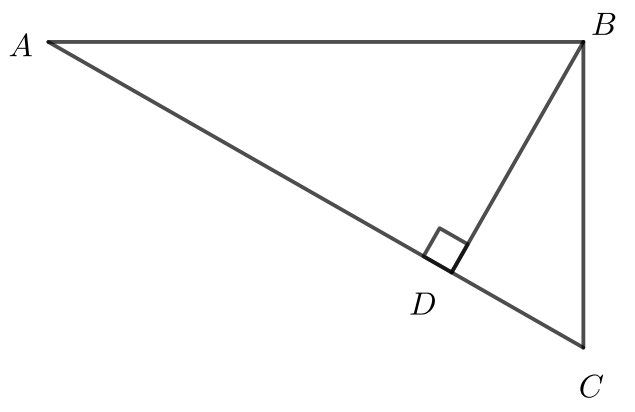

Fig. 1: This is an Euclidean right triangle. Euclidean geometry is the geometry which areas, angles and lengths are related together by whole and certain numbers and there are no complex, uncertain or irrational numbers, irrational numbers are with a limit. Instead of this right triangle, it can be used a triangle which there is no right angle occurs in it. The shape is random. It does not mean actual geometry. For example, $\mathrm{BD}$ length can be longer than $\mathrm{AD}$ length in the below stated calculations.

There is a representation of an Euclidean geometry. In this case, being $\mathrm{BD}$ and $\mathrm{BC}$ are fixed, assume that $\mathrm{AB}$ is lengthened to any other $\mathrm{AB}_{2}$ length. Here $A$ point can be assumed as moving body in free space, $\mathrm{B}$ point is a fixed point which its coordinate is known in space and $\mathrm{C}$ is an observer.

For the lengthened hypotenuse on Fig. 1, over the inequality and definition of $\mathrm{AB}_{2}>\mathrm{AB}$, it becomes (1) over the inequality of $\mathrm{BD}_{2}^{2}-\mathrm{BD}^{2}>\mathrm{AD}^{2}-\mathrm{AD}_{2}^{2}$,

$$
1>\frac{\mathrm{AD}^{2}-\mathrm{AD}_{2}^{2}}{\mathrm{BD}_{2}^{2}-\mathrm{BD}^{2}}
$$

where $\mathrm{BD}^{2}+\mathrm{AD}^{2}=\mathrm{AB}^{2}$ and $\mathrm{BD}_{2}^{2}+\mathrm{AD}_{2}^{2}=\mathrm{AB}_{2}^{2}$ are the equations over Pythagorean theorem. In the same manner, it becomes (2),

$$
\mathrm{BD}^{2}+\mathrm{DC}^{2}=\mathrm{BD}_{2}^{2}+\mathrm{DC}_{2}^{2}
$$

where $\mathrm{h}^{2}+\mathrm{t}^{2}=\mathrm{y}^{2}$ and $\mathrm{h}_{2}^{2}+\mathrm{t}_{2}^{2}=\mathrm{y}^{2}$ are the equations over Pythagorean theorem. If (2) is edited, it becomes $\mathrm{DC}^{2}$ $\mathrm{DC}_{2}^{2}=\mathrm{BD}_{2}^{2}-\mathrm{BD}^{2}$; thus if $\mathrm{DC}^{2}-\mathrm{DC}_{2}^{2}$ is used instead of $\mathrm{BD}_{2}^{2}-\mathrm{BD}^{2}$ on (1), also it becomes (3) over $\mathrm{DC}^{2}-\mathrm{DC}_{2}^{2}>$ $\mathrm{AD}^{2}-\mathrm{AD}_{2}^{2}$ inequality.

$$
1>\frac{\mathrm{AD}^{2}-\mathrm{AD}_{2}^{2}}{\mathrm{DC}^{2}-\mathrm{DC}_{2}^{2}}
$$

Now the actual displacement inequalities have been determined. Right this point, assume that there is no displacement, namely there is no lengthening. For this condition, it becomes $\mathrm{AB}_{2}=0, \mathrm{DC}_{2}=0$ and $\mathrm{AD}_{2}=0$; thus (3) becomes (4),

$$
\mathrm{DC}^{2}>\mathrm{AD}^{2}
$$

and (1) becomes (5).

$$
-\mathrm{BD}^{2}>\mathrm{AD}^{2}
$$

\section{Some results of the inequality}

The inequality of (5) actually means it is impossible to be $\mathrm{AD}=\mathrm{DC}$ that means perpendicularity is impossible in universe. This also means that at the same time no lengths can be the same. From physical perspective, it means each point of free space has the same speed and energy magnitude at the end of 1 second but the same time, and emergence is one by one for the total of universe. Each point emerges by order. This also means, for any force-applied, since there is no middle point the natural motion is always circular as there is no alternative. Because of circular displacement, centrifugal force is always together with motion.

The inequality of (5) has another results, and are as the below stated ones.

- Exactly there is no middle point place.

- A right angle cannot emerge. It is only close to right angle due to the energy which area holds.

- It cannot be drawn two line segments which have the same length from a point in space to other two points. Namely, 3 or more objects cannot take place in space being the distance between each of them is the same. There is a time difference between each point of space at the same assumed global time.

- For $\mathrm{AD} \neq \mathrm{DC}$, it also becomes $\mathrm{AB} \neq \mathrm{BC}, \mathrm{BD} \neq \mathrm{DC}$, $\mathrm{BD} \neq \mathrm{AD}, \mathrm{BD} \neq \mathrm{AB}$ and more. Namely, while $\mathrm{AB}$ is lengthened, $\mathrm{BC}$ or the other lengths cannot protect own actual length. The medium is conservative. This also means, that length and thus 1 dimension do not 
exist alone; because 2 objects cannot take place at a distance relatively to each other. Namely, length is not absolute since is relative. Length occurs in a limited time interval and gets lost constantly, and cannot be independent on speed. Higher dimension parts of lower dimensions have different size than lower dimensional parts. Namely a 2 dimensional square cannot be used by the same size to create a 3 dimensional cube.

- The shortest distance between 2 objects is not a line segment. This distance is an arc so close to a line segment.

- Parallel two line segments cannot be drawn beyond drawing line segment. They are exactly intersected, and the intersection point occurs due to area multitude of conservative area.

- A closed curve is not possible. Only infinite space closes curve. Limited space is not closed but is conservative.

\section{Magic of number world}

Since in a conservative limited area no two lengths can be equal to each other, the numbers which are defined as $\mathrm{x}^{\mathrm{y}} \cdot \mathrm{z}$ where $\mathrm{x}, \mathrm{y}, \mathrm{z} \in \mathbb{N}^{+}$are not real numbers. These are imaginary numbers since are also formed by two the same numbers. Namely, the numbers like 4,8,12,16 and 25 are not real numbers. Even if they can be obtained by addition, multiplication is a process alone as a phenomenon which has different, peculiar and exact properties. You cannot use always addition and subtraction instead of multiplication and division to achieve the same number. Namely, you cannot realize verifying always that this means if a number cannot be obtained by multiplication, then it means also addition will be meaningless. This is very important especially for physics; because in real physical medium it has different meanings. For example fine-tuning problem may occur because of this. While we are using some series to calculate something, we may ignore something important.

Even if we eliminate some numbers from the number line, still there do not exist only prime numbers. If there were only prime numbers, it would mean that the equality is always $\mathrm{P}=\mathrm{NP}$ since there is no prime factors, and would mean that each length of a polygon or a shape in the universe would get different side lengths as prime numbers that means the universe would be formed only by primes. Even so, still each length of a polygon has different side lengths that some of them are exactly prime numbers.

\section{First solution to the $\mathbf{P}=\mathrm{NP}$ ? problem}

Over the above stated information, first of all we can stated that as it was said as the above, higher dimensional parts have different sizes. It means that to create a 3 dimensional cube, if you have a 2 dimensional square which has different side lengths that has A energy in real physical medium, you must use a different square which has B energy since has different side lengths in itself and has different lengths than the other one where $\mathrm{A}<\mathrm{B}$ or $\mathrm{A}>\mathrm{B}$. It means to create higher numbers or higher dimensions you must use more energy. You can say that higher dimensions can multiply by getting smaller in size forever as also can get bigger; but it does not differ since you can go from the reverse direction. It also means, if a number is formed by multiplication of different prime numbers, you must use more energy to find the prime factors even if you assumed that you know the places where the prime numbers are placed there. It can be maintained as the following one.

The state of $\mathrm{P}=\mathrm{NP}$ is only dream.

Proposition X

\section{Second solution to the $\mathrm{P}=\mathrm{NP}$ ? problem}

An additional supportive solution can be realized as well. In the set of odd numbers, assume that there are prime numbers where odd multiples of 3 are placed instead of odd multiples of 3 . In this case, also assume that the numbers inbetween are non-prime numbers.

$$
\mathrm{N}_{\mathrm{A} 1}\left|\mathrm{~N}_{\mathrm{B} 1}\right| \mathbf{9}\left|\mathrm{N}_{\mathrm{A} 2}\right| \mathrm{N}_{\mathrm{B} 2}|\mathbf{1 5}| \mathrm{N}_{\mathrm{A} 3}\left|\mathrm{~N}_{\mathrm{B} 3}\right| \mathbf{2 1}
$$

Table 1: Distribution of odd multiples of 3 for a limited interval. Here $\mathrm{N}_{\mathrm{A}}$ numbers are different non-prime numbers which occur after odd multiples of 3 , and $\mathrm{N}_{\mathrm{B}}$ numbers are different non-prime numbers occur before odd multiples of 3 since there are only 2 odd numbers between each consecutive odd multiples of 3 in the set of odd numbers.

Here is a table which was created over this assumption. Here we must ask that in the real set of odd numbers which is independent of the table, for a selected odd multiple of 3 , are there equal number of prime numbers to the number of odd numbers between 3 and the selected odd multiple of 3 . If the answer is yes, then in the set of odd numbers between two consecutive odd multiple of 3 , there must exist minimum 1 prime number; but this is impossible since there always must be non-prime twins. For example even if we can choose infinite different combination, we can choose odd multiples of 5 and 7 as non-prime twins. Distribution of 5 in the set of odd numbers is over $f(x)=10 x-5$ function as 7 is over $f(y)=14 y-7$ as well; so as the difference between two consecutive multiple of 5 and 7 as twin is going to be 2 , the equation must be $f(x)=f(y)+2$. Over this equation it must be (6) where $x, y \in \mathbb{N}^{+}$

$$
5 \mathrm{x}=7 \mathrm{y}
$$

Here for each $\mathrm{x}$ and $\mathrm{y}$ values which provides the equality, there are infinite number of non-prime twins. Examples can be applied for any numbers like this equation. This is not only valid for 5 and 7.

This means that for a selected odd multiple of 3 there is not exactly 1 prime number between each consecutive odd multiples of 3 until the selected odd number. Namely number of non-prime numbers are more than prime numbers until the selected odd multiple of 3 . In this case, if we assume that there exist prime numbers in the places which odd multiples of 3 exist instead of odd multiples of 3 , then this means for the selected odd multiple of 3, non-prime numbers are going to be more than prime numbers due to the table. This increases possibility of to be non-prime number of a selected number in the set of odd number over the assumption and thus also increases prime factor processes since also more non-primes exist due to the assumption for a selected number. Numbers are formed by more non-prime numbers as unusual as also number of prime numbers are decreased by this assumption. This is for the worst possibility. Namely if we prove over this assumption that for a selected number, the process number for primality test is not equal to the process number for finding out prime factors, no other proofs are required since is the worst possibility.

In this case, over the above stated information now the function which gives each prime is known as $f(x)=6 x-3$ function since the function is distribution rule of odd multiples of 3 as primes are counted as exist at the places of these multiples as well.

As a result there is a function which test primality as (7) over $f(x)=6 x-3$. 


$$
\mathrm{f}^{-1}(\mathrm{x})=\frac{\mathrm{x}+3}{6}
$$

If result is a whole number, then the number is prime, otherwise is not prime. If is not prime, then minimum $\left\lfloor\frac{x+3}{6}\right\rfloor$ process number requires that it means always for primality test of a number, less process requires than finding out prime factors.

For the worst possibility, the state of $\mathrm{P}=\mathrm{NP}$ is completely dream.

\section{Proposition Y}

As a result, $\mathrm{P}=\mathrm{NP}$ state is not possible that only 1 evidence is enough since assumed function which provides $\mathrm{P}=\mathrm{NP}$ state must also work for this primality and prime factor problem. Even if the above stated solution is not going to work for some numbers that especially at the beginning of the set of odd numbers, even only 1 number in the set of odd number can be counted as evidence. We must accept the set of odd numbers as infinite not in an interval. Already, in the infinite set, logically non-primes can be accepted as more than primes since when a new prime emerges, it is combined with the early emerging ones to create more number. This creates many possibilities and thus creates many combinations.

\section{Progressive image}

There is a special condition in the above stated first solution to $\mathrm{P}=\mathrm{NP}$ ? problem that if you accept the twin which has more energy of higher dimensional polygon and thus numbers as smaller numbers, then an excessive work emerges. It means, the problems which are not dependent on a single polynomial as NP problems, can be solved easier; but it requires soothsaying since information is deterministic. Below stated information proves this over the above stated rules of the triangle of Fig. 1.

For the lengthened hypotenuse of Fig. 1, over the inequality of $\left(\mathrm{AD}_{2}+\mathrm{DC}_{2}\right)^{2}>(\mathrm{AD}+\mathrm{DC})^{2}$ where $\mathrm{AB}_{2}^{2}+\mathrm{BC}_{2}^{2}=$ $\left(\mathrm{AD}_{2}+\mathrm{DC}_{2}\right)^{2}$ and $\mathrm{AB}^{2}+\mathrm{BC}^{2}=(\mathrm{AD}+\mathrm{DC})^{2}$, it becomes (8) for $\mathrm{AB}_{2}=0$ that means no lengthening.

$$
0>(\mathrm{AD}+\mathrm{DC})^{2}
$$

It means, that $\mathrm{AD}+\mathrm{DC} \notin \mathbb{R}$. If you assume, that $\mathrm{AD}+$ $\mathrm{DC}=0$, it becomes $0>0$; thus actually non of them can be 0 . It means, that even if there would be no lengthening and thus no motion, there were already area and motion. They are deterministic and cannot be $0 . \mathrm{AD}+\mathrm{DC}$ is always an imaginary number for the condition of (8).

Area and thus all the information which area holds are deterministic. They cannot be created afterwards.

\section{Proposition $\mathbf{Z}$}

In accordance with conservative space, over the components of the right triangle, it becomes $\mathrm{AB}+\mathrm{BC}+\mathrm{m}=\mathrm{n}$ and $\mathrm{AB}^{2}+\mathrm{BC}^{2}=\mathrm{m}^{2}$ where $\mathrm{m}=\mathrm{AD}+\mathrm{DC}$; thus becomes $\tau+\mathrm{DC}+\mathrm{m}=\mathrm{n}$ and $\tau^{2}+\mathrm{t}^{2}=\mathrm{m}^{2}$ where $\tau$ and $\mathrm{t}$ are time here, and is $\mathrm{m}=\sqrt{\tau^{2}+\mathrm{t}^{2}}$. It seems, that $\mathrm{AB}$ and $\mathrm{BC}$ or $\tau$ and t cannot take random values, that they take certain values in accordance with a rule. If $\tau+\mathrm{t}+\mathrm{m}=\mathrm{n}$ is edited, it becomes $(\tau+\mathrm{t})^{2}=(\mathrm{n}-\mathrm{m})^{2}$ and thus it becomes $\tau^{2}+\mathrm{t}^{2}+2 \tau \mathrm{t}=\mathrm{n}^{2}-2 \mathrm{~nm}+\mathrm{m}^{2}$. Since it is $\tau^{2}+\mathrm{t}^{2}=\mathrm{m}^{2}$, finally it becomes (9),

$$
2(\tau \mathrm{t}-\mathrm{nm})-\mathrm{n}^{2}=0
$$

where $n=\tau+t+\sqrt{\tau^{2}+t^{2}}$. For this equation of (9), the roots become (10) as imaginary time since it cannot be 0 over (8).

$$
\tau=\text { 干it }
$$

With a motion, information in the imaginary time emerges in our universe. As constant speed and so $\mathrm{x}=\mathrm{vt}$ is not possible, because of $\mathrm{x}=\mathrm{at}^{2}$, when real time is emerged over $\mathrm{t}^{2}$ in $\mathrm{x}=\mathrm{at}^{2}$ by using imaginary time of (10) instead of the $\mathrm{t}^{2}$, the distance and time emerge in opposite ways over the time on $\mathrm{x}=\mathrm{at}^{2}$, and know, that image appears as illusion in $3 \mathrm{D}$ because of the complex roots and thus complex plane which is perpendicular to (x,y) plane. After that, if you want to express the motion as average, you do not have to use imaginary time since is real time after this, just use its multitude on $\mathrm{x}=\mathrm{vt}$ and it becomes $\mathrm{x}=-\mathrm{vt}$. Excessive information is in the imaginary time that this information is result of any problem. You do not make process for NP problem. You only realize process to learn the deterministic result which had already been existed. It also is not related with quantum computers. They do not realize soothsaying.

\section{Acknowledgment}

This is it! Goodbye!

\section{References}

1. Kavak M. 2018, Complementary Inferences on Theoretical Physics and Mathematics, OSF Preprints. Available online: https://osf.io/tw52w/ 